\title{
Traveltime and amplitude calculations using the damped wave solution
}

\author{
Changsoo Shin*, Dong-Joo Min $\ddagger$, Kurt J. Marfurt**, Harry Y. Lim*, \\ Dongwoo Yang*, Youngho Cha*, Seungwon Ko*, Kwangjin Yoon*, \\ Taeyoung $\mathrm{Ha}^{*}$, and Soonduk Hong*
}

\begin{abstract}
Because of its computational efficiency, prestack Kirchhoff depth migration remains the method of choice for all but the most complicated geological depth structures. Further improvement in computational speed and amplitude estimation will allow us to use such technology more routinely and generate better images. To this end, we developed a new, accurate, and economical algorithm to calculate first-arrival traveltimes and amplitudes for an arbitrarily complex earth model. Our method is based on numerical solutions of the wave equation obtained by using well-established finite-difference or finite-element modeling algorithms in the Laplace domain, where a damping term is naturally incorporated in the wave equation. We show that solving the strongly damped wave equation is equivalent to solving the eikonal and transport equations simultaneously at a fixed reference frequency, which properly accounts for caustics and other problems encountered in ray theory. Using our algorithm, we can easily calculate first-arrival traveltimes for given models. We present numerical examples for 2-D acoustic models having irregular topography and complex geological structure using a finite-element modeling code.
\end{abstract}

\section{INTRODUCTION}

Efficient and accurate traveltime and amplitude calculation from surface to depth points is of critical importance in Kirchhoff prestack depth migration as well as to transmission and refraction tomography.

Ray-tracing techniques have widely been used for traveltime calculation. Shooting-ray (Cerveny et al., 1977) and ray- bending methods (Thurber and Ellsworth, 1980) form the basis for most commonly used algorithms. Both of these conventional ray-tracing methods can become quite expensive if we calculate traveltimes at finely spaced angles and then interpolate the traveltimes onto a regular grid. In addition, ray methods often suffer from shadow zones and local minima (Coultrip, 1993).

Vidale (1988) suggested a method which directly computes first-arrival traveltimes on a regular grid by calculating finitedifference solutions of the eikonal equation. Van Trier and Symes (1991) vectorized Vidale's algorithm, and Qin et al. (1992) improved its stability by using expanding wavefronts. Podvin and Lecomte (1991) further improved the eikonal solver to reduce significant traveltime errors for models with a high velocity contrast. Building upon Podvin and Lecomte's (1991) work, Schneider et al. (1992) calculated eight traveltimes for eight propagation ranges of $45^{\circ}$ at each grid point, from which they extracted the minimum traveltime. This approach is easily applicable to anisotropic problems. Ettrich and Gajewski (1998) suggested a method which calculates traveltimes for an initial velocity model using Vidale's (1988) method and then computes traveltimes for slightly different velocity models using a finite-difference perturbation method. This perturbation method is easily adapted to computing traveltimes for weakly anisotropic media.

Coultrip (1993) and Vinje et al. (1993) computed traveltimes using a wavefront construction algorithm and showed that wavefront construction is easily applied to prestack depth migration. Nichols (1996) presented a method which calculates traveltimes and amplitudes in the seismic frequency band by solving the one-way wave equation in the frequency domain. By assuming that the phase of the seismic signal varies linearly with frequency within a narrow seismic band, Nichols (1996) was able to obtain both traveltimes and amplitudes of maximum energy arrivals. Shin et al. (2000) also devised a traveltime calculation algorithm using finite-difference solutions

Published on Geophysics Online June 24, 2002. Manuscript received by the Editor December 4, 2000; revised manuscript received March 26, 2002. *Seoul National University, School of Civil, Urban \& Geosystem Engineering, San 56-1, Sillim-dong, Kwanak-Ku, Seoul, 151-740, Korea. E-mail: css@model.snu.ac.kr; yhcha@gpl.snu.ac.kr; gplko@msn.com; tyha@gpl.snu.ac.kr; sdhongl@yahoo.co.kr.

†Korea Ocean Research \& Development Institute, Ansan, Post Office Box 29, Kyungki, 425-600, Korea. E-mail: djmin@kordi.re.kr.

**University of Houston, Allied Geophysical Laboratories, Department of Geoscience, Houston, Texas 77204-5006. E-mail: kmarfurt@uh.edu.

(C) 2002 Society of Exploration Geophysicists. All rights reserved. 
of the one-way wave equation using a few seismic frequencies. They introduced complex frequencies commonly used to suppress wraparound in the frequency-domain modeling algorithms. By choosing this wraparound suppression factor to be sufficiently large, they were able to suppress all the energy (signal and noise) following the first arrival. In addition, by using the one-way wave equation, their technique favors traveltimes of direct arrivals rather than upcoming head waves.

The most accurate and reliable technique for determining traveltime and amplitude is to compute the full hyperbolic wavefield using either the finite-difference, finite-element, or pseudospectral methods at a relatively high frequency and then pick the traveltime and amplitude of each wave event. Unfortunately, this method is quite expensive, even for 2-D velocity models.

In this paper, we approximate a wavefield by a series of weighted spikes. By solving the wave equation in the Laplace domain, where the wave equation automatically includes a damping term, we can suppress all the events following the first-arrival event. We begin our discussion by showing that a solution of the wave equation in the Laplace domain can be expressed as a series of damped spikes. Next, we show how to calculate traveltimes and amplitudes from the solutions of the Laplace-transformed wave equation at a single Laplace frequency. Finally, we present some numerical examples for a suite of 2-D acoustic models with irregular topography and complex geological structure.

\section{METHODOLOGY}

\section{Approximating wavefields by amplitude and traveltime}

We assume that a seismic signal observed at a receiver in depth can be approximated by a series of weighted spikes (Figure 1). The weighted series of spikes can be expressed as

$$
u(t)=\sum_{n} A_{n} \delta\left(t-t_{n}\right)
$$

where $A_{n}$ and $t_{n}$ are the amplitude and the $n$th digitized time (counted from the first-arrival event), respectively. In general, if we multiply equation (1) by a strong damping factor $e^{-\alpha t}$, we can suppress all the events following the first arrival, as shown in Figure 2, and thus approximate the solution as

$$
u^{*}(t)=u(t) e^{-\alpha t} \cong A_{1} e^{-\alpha t} \delta\left(t-t_{1}\right),
$$

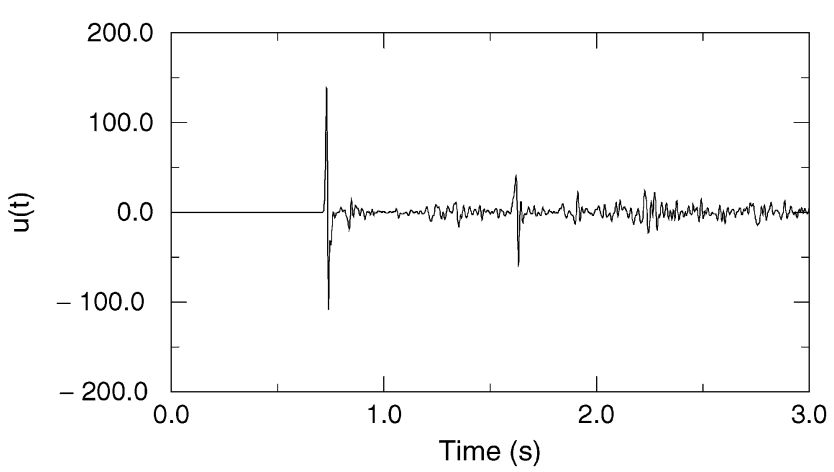

FIG. 1. A synthetic seismogram for a 2-D earth model. The seismic signal can be approximated by a series of weighted spikes. where $A_{1}$ and $t_{1}$ are the amplitude and the traveltime of the first arrival. By suppressing all the wave events following the first arrival, we transform the difficult first-arrival picking problem into the easier maximum-arrival picking problem.

\section{Wave equation in the Laplace domain}

When we solve the wave equation using finite-element methods, we obtain

$$
\mathbf{M u ̈}+\mathbf{K u}=\mathbf{f},
$$

where $\mathbf{M}$ is the mass matrix, $\mathbf{K}$ is the stiffness matrix, $\mathbf{u}$ is the unknown wavefield, $\ddot{\mathbf{i}}$ is the second-order time derivative of $\mathbf{u}$, and $\mathbf{f}$ is the source vector (Marfurt, 1984).

Taking the Laplace transform of equation (3) yields

$$
\mathbf{S} \tilde{\mathbf{U}}=\tilde{\mathbf{F}},
$$

with

$$
\begin{gathered}
\mathbf{S}=\mathbf{M} s^{2}+\mathbf{K}, \\
\tilde{\mathbf{U}}=\int_{0}^{\infty} \mathbf{u}(t) e^{-s t} d t,
\end{gathered}
$$

and

$$
\tilde{\mathbf{F}}=\int_{0}^{\infty} \mathbf{f}(t) e^{-s t} d t,
$$

where $s$ is the Laplace frequency. In order to obtain the wavefield $\tilde{\mathbf{U}}$ in the Laplace domain, we factor the real impedance matrix $\mathbf{S}$ into an upper and a lower triangular matrix, using either Doolittle's, Crout's, or Cholesky's method (Kreyszig, 1993). We next obtain the wavefield $\tilde{\mathbf{U}}$ in the Laplace domain using a simple forward and backward substitution.

Note that the integrand of equation (6) is a damped signal like $u^{*}$ in equation (2). Hence, treating $u$ and $A_{n}$ as column vectors, we note that by substituting $u$ in equations (1) and (2) for $\mathbf{u}$ in equation (6), we can write the Laplace-transformed wavefield for a relatively high Laplace frequency $s$ as

$$
\tilde{\mathbf{U}}=\sum_{n} \mathbf{A}_{n}(x, z) e^{-s \mathbf{t}_{n}(x, z)} \cong \mathbf{A}_{1}(x, z) e^{-s \mathbf{t}_{1}(x, z)} .
$$

By using the wavefield $\tilde{\mathbf{U}}$ and the derivative wavefield $(\partial \tilde{\mathbf{U}} / \partial s)$ at a single Laplace frequency $s$ in the Laplace domain, we will demonstrate how to efficiently calculate traveltime and amplitude of the first-arrival event.

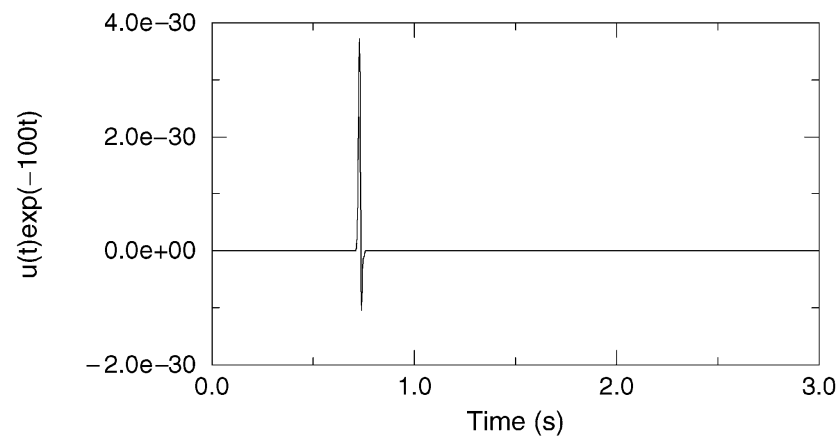

FIG. 2. A delta-like wavefield obtained by introducing a strong damping factor $e^{-100 t}$. 


\section{HOW TO CALCULATE TRAVELTIMES AND AMPLITUDES}

If we take the derivative of equation (8) with respect to $s$, we obtain

$$
\frac{\partial \tilde{\mathbf{U}}}{\partial s}=-\mathbf{t}_{1}(x, z) \mathbf{A}_{1}(x, z) e^{-s \mathbf{t}_{1}(x, z)}=-\mathbf{t}_{1}(x, z) \tilde{\mathbf{U}} .
$$

From equations (8) and (9), we calculate the traveltime $\tau(x, z)$ and amplitude $\mathbf{A}_{1}(x, z)$ :

$$
\mathbf{t}_{1}(x, z)=\frac{-1}{\tilde{\mathbf{U}}} \frac{\partial \tilde{\mathbf{U}}}{\partial s}
$$

and

$$
\mathbf{A}_{1}(x, z)=\tilde{\mathbf{U}} e^{s \mathbf{t}_{1}(x, z)} .
$$

We explain how to efficiently compute $(\partial \tilde{\mathbf{U}} / \partial s)$ in Appendix A. We call our method suppressed wave equation estimation of traveltime (SWEET). In Appendix B, we show how our SWEET method is equivalent to simultaneously solving the eikonal and transport equations. In particular, if we substitute equation (8) into the wave equation in the high Laplace frequency limit, we will obtain the eikonal equation coupled with the transport equation.

\section{AN OPTIMUM LAPLACE FREQUENCY}

Correct traveltimes requires proper selection of the Laplace frequency. A large Laplace frequency strongly damps all the wavefield except the first-arrival event, so that we can easily pick the first-arrival traveltimes. However, a large Laplace frequency also requires a fine grid to minimize numerical dispersion, resulting in increased computational cost. A small Laplace frequency results in economic calculations, but the small Laplace frequency may introduce errors in picking our first arrival because it does not completely damp wave events following the first arrival. For this reason, we need to optimize our choice of Laplace frequency so that we minimize dispersions and damp all the wave events following the first-arrival event.

In order to do so, we generalize work by Marfurt (1984) to analyze the dispersion relation of the Laplace-transformed wave equation. The dispersion analysis is in general performed by substituting plane-wave solutions for a homogeneous medium into a discrete form of the wave equation and computing normalized, numerical phase velocities as a function of frequency and angle. In the frequency domain, the plane-wave solutions are the same as the eigenfunctions of the
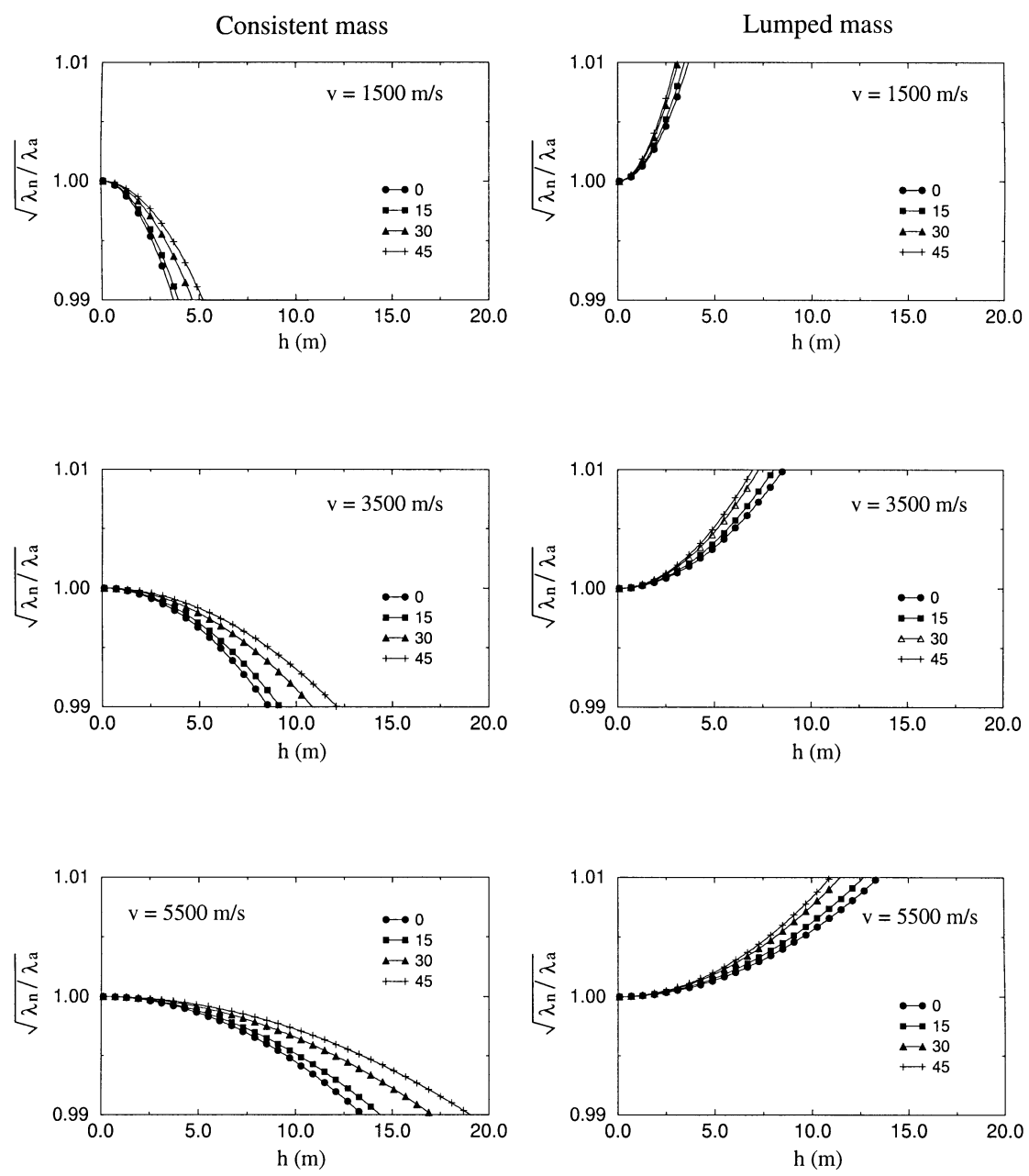

FIG. 3. Dispersion curves for the consistent (left) and the lumped (right) mass-matrix operator when velocities are $1500 \mathrm{~m} / \mathrm{s}$ (upper), $3500 \mathrm{~m} / \mathrm{s}$ (middle), and $5500 \mathrm{~m} / \mathrm{s}$ (bottom), respectively, and the Laplace frequency is 200 . The parameters $\lambda_{n}$ and $\lambda_{a}$ are the numerical and analytic eigenvalues, respectively. 
Laplace equation $\left(v^{2} \nabla^{2} u=0\right)$. By introducing the concept of eigenfunctions, the dispersion relation can be expressed as the square root of the ratio of the numerical eigenvalue to the analytic eigenvalue $\left(\sqrt{\lambda_{n} / \lambda_{a}}\right.$; where $\lambda_{n}$ and $\lambda_{a}$ are the numerical and analytic eigenvalues, respectively). In the Laplace domain, we obtain the dispersion relation by using the eigenfunction rather than the plane-wave solution, because we cannot readily define wavenumber, wavelength, and plane-wave solutions (Appendix C). The dispersion relation of the Laplacetransformed wave equation is analogous to the frequencydomain wave equation.

Once we obtain our dispersion relation, we could determine the grid interval and the optimum Laplace frequency which will be used to compute traveltimes and amplitudes for a given velocity model by analyzing dispersion curves for various Laplace frequencies, grid intervals, velocities, and wave-propagation angles. From the dispersion curves, we can also extract the empirical relationship between grid interval, velocity, and Laplace frequency. The relationship has a form analogous to that of the more familiar frequency-domain wave equation:

$$
s_{\text {optimum }}=\frac{2 \pi v_{\mathrm{ave}}}{G^{\prime} \Delta},
$$

where $s_{\text {optimum }}$ is the optimal Laplace frequency corresponding to angular frequency $\omega, v_{\text {ave }}$ is the average velocity of a given model, $\Delta$ is the grid interval, and $G^{\prime}$ is the number of grid points per pseudowavelength (i.e., the equivalent to wavelength in the frequency domain). Since we do not actually know how to define wavelength in the Laplace domain, we define the equivalent concept to wavelength in the frequency domain as pseudowavelength in the Laplace domain.

The characteristic of dispersion curves for the finite-element method in the frequency domain is dependent upon a means of constructing the mass matrix (Marfurt, 1984). We compute dispersion curves for the two conventional finite-element methods using the consistent and lumped mass-matrix operators (e.g., Marfurt, 1984; Jo et al., 1996). When using the consistent massmatrix operator to approximate the mass term, the square root of normalized eigenvalue $\left(\sqrt{\lambda_{n} / \lambda_{a}}\right)$ decreases as $G^{\prime}$ decreases (Figure 3, left). On the other hand, the dispersion relation for

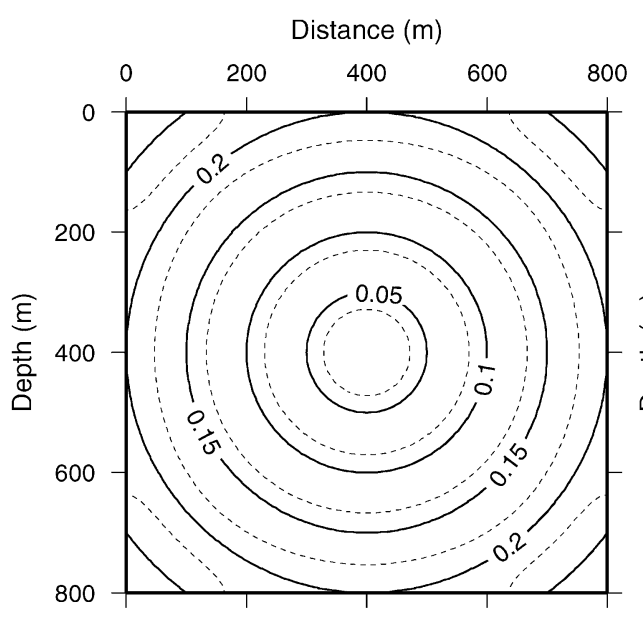

(a)

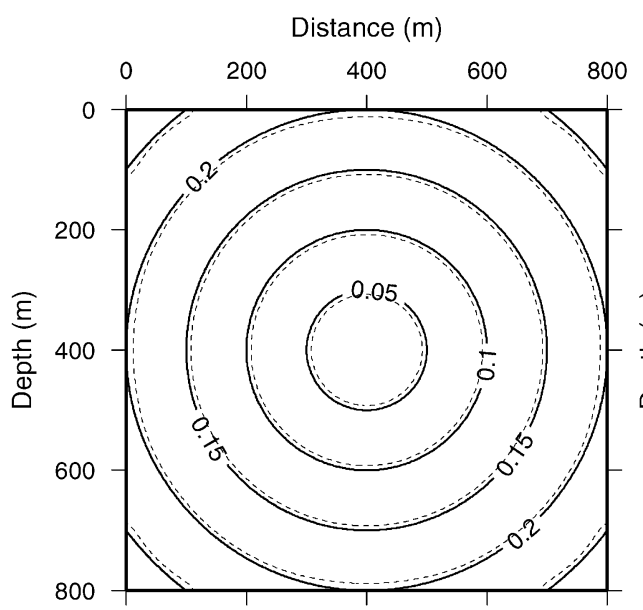

(c)

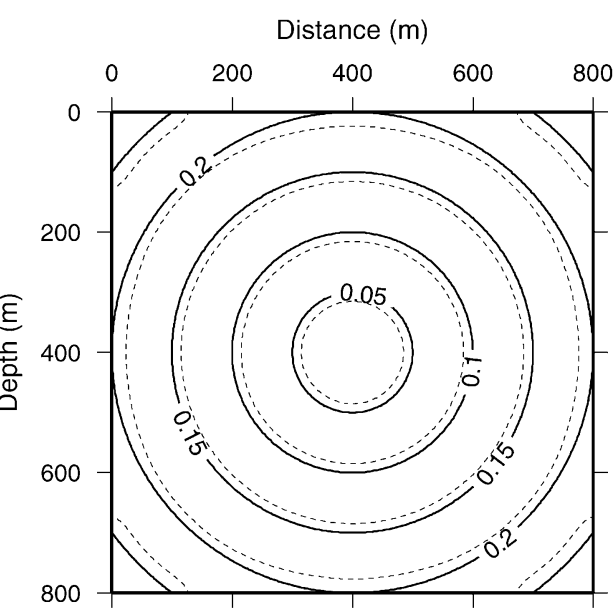

(b)

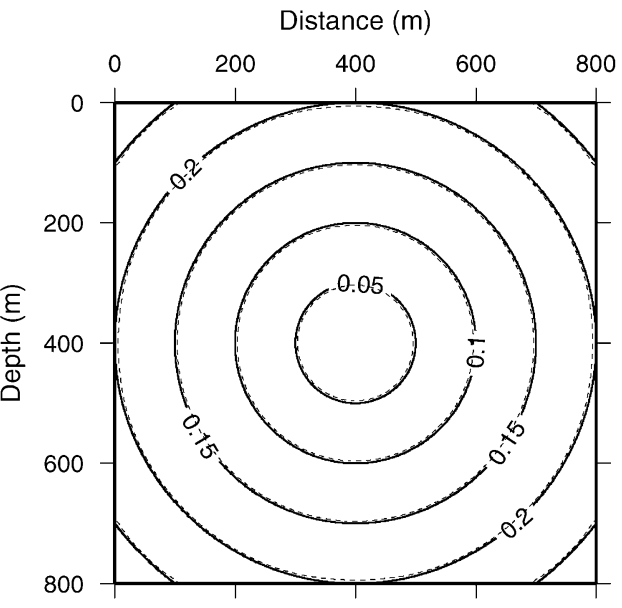

(d)

FIG. 4. Analytic traveltimes (solid lines) and numerical traveltimes (dotted lines) computed by the SWEET method for a homogeneous model whose size is $800 \mathrm{~m} \times 800 \mathrm{~m}$, when (a) $s=10 \pi, \Delta=8 \mathrm{~m}$; (b) $s=20 \pi, \Delta=4 \mathrm{~m}$; (c) $s=40 \pi, \Delta=2 \mathrm{~m}$; and (d) $s=80 \pi, \Delta=1 \mathrm{~m}$. A source is located at the center of the model. 
the lumped mass-matrix operator has the opposite characteristic (Figure 3, right). For simplicity of programing computer code, we will use the lumped mass-matrix operator for traveltime and amplitude computation. For the lumped mass-matrix operator, the number of grid points per pseudowavelength $\left(G^{\prime}=25\right)$ is determined by analyzing dispersion curves having errors less than $0.4 \%$.

\section{A RELATIONSHIP BETWEEN LAPLACE FREQUENCY AND TRAVELTIME ERROR}

We compute traveltime errors for a homogeneous model as a function of the Laplace frequency. We begin by taking the homogeneous model $(800 \mathrm{~m} \times 800 \mathrm{~m})$ with a constant velocity of $2000 \mathrm{~m} / \mathrm{s}$ and apply a source at the center of the model. We then analyze traveltime errors for two cases: one where both the Laplace frequency and grid interval change, and the other where the Laplace frequency changes but the grid interval remains fixed.

In the first analysis, we use Laplace frequencies of $10 \pi, 20 \pi$, $40 \pi$, and $80 \pi$, where the grid intervals determined with $G^{\prime}=50$ in equation (12) are $8 \mathrm{~m}, 4 \mathrm{~m}, 2 \mathrm{~m}$, and $1 \mathrm{~m}$, respectively. In Figure 4, we display analytic traveltimes and numerical traveltimes computed by the SWEET method for the homogeneous model. In Figures 4a, 4b, and 4c, we note that edge reflections generated from the finite-size model interfere with our ability to choose the first arrival. In Figure 4d, we note that numerical traveltimes agree well with analytic traveltimes in the entire model. The accuracy of our numerical traveltimes increases with Laplace frequency.

In the second analysis, we compare analytic traveltimes with numerical traveltimes obtained with a fixed grid interval of $2 \mathrm{~m}$ for the same model. We also use the same Laplace frequencies. For the Laplace frequencies of $10 \pi, 20 \pi$, and $40 \pi$, the numerical dispersion errors are less than $0.1 \%$, whereas for the Laplace frequency of $80 \pi$, the numerical dispersion errors are no larger than $0.4 \%$ with $G^{\prime}=25$. In Figures 5a, 5b, and $5 \mathrm{c}$, we can note that numerical traveltimes are the same as those in Figures $4 a, 4 b$, and $4 c$, whereas the numerical traveltimes with the error of $0.4 \%$ in Figure $5 \mathrm{~d}$ are more compatible with analytic traveltimes than those with the error of $0.1 \%$ in Figure 4. Our numerical tests tell us that we can obtain the most

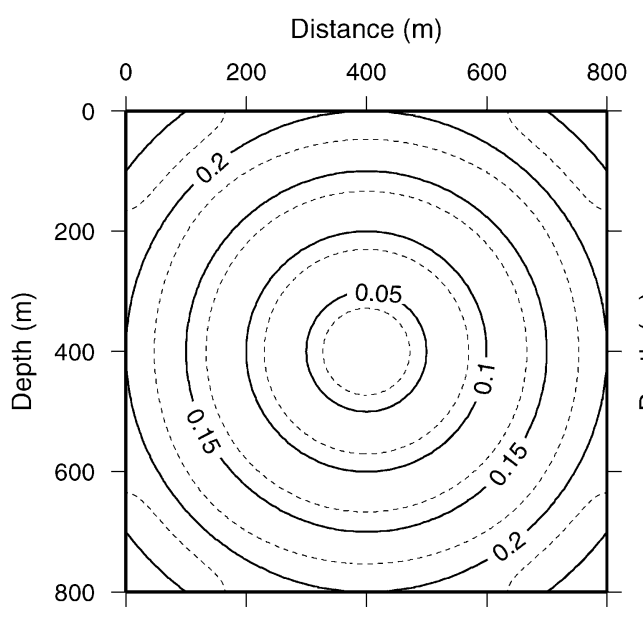

(a)

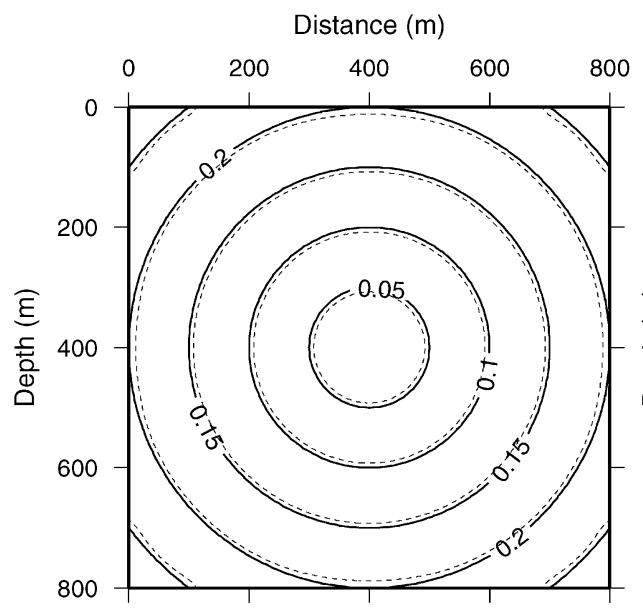

(c)

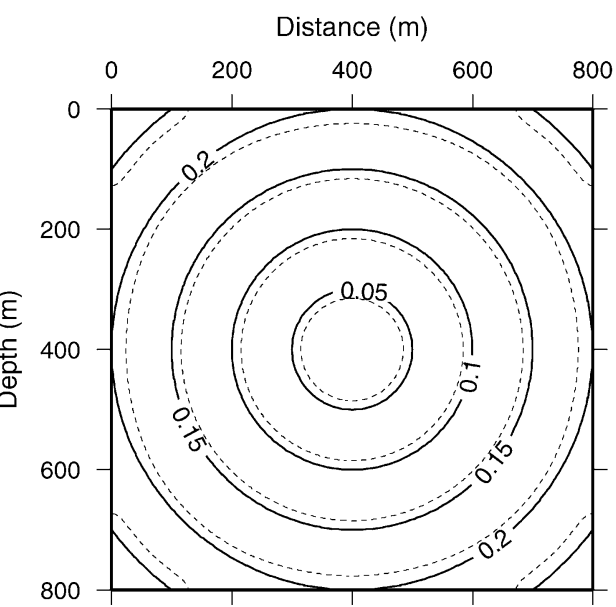

(b)

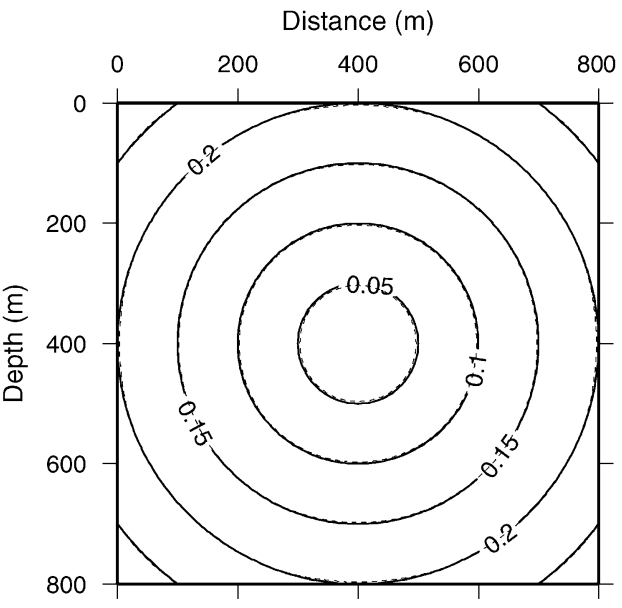

(d)

FIG. 5. Analytic traveltimes (solid lines) and numerical traveltimes (dotted lines) computed by the SWEET method with a fixed grid interval of $2 \mathrm{~m}$ for a homogeneous model whose size is $800 \mathrm{~m} \times 800 \mathrm{~m}$, when (a) $s=10 \pi$, (b) $s=20 \pi$, (c) $s=40 \pi$, and (d) $s=80 \pi$. A source is located at the center of the model. 
accurate traveltimes when we choose the number of grid points per pseudowavelength to be $G^{\prime}=25$.

\section{COMPUTATIONAL EFFICIENCY}

The computational effort required to generate traveltime and amplitude tables using conventional ray-tracing techniques is linearly proportional to the number of shots. In our SWEET algorithm, once we factor the impedance matrix, the cost also increases in proportion to the number of shot points. Even though our SWEET algorithm requires an extra cost to decompose the symmetric impedance matrix, the total cost for our algorithm is comparable to that of conventional ray-tracing methods.

\section{NUMERICAL EXAMPLES}

In order to verify our SWEET solution, we first examine a two-layer model with a local low-velocity zone similar to that presented by Vinje et al. (1993). To show the robustness of the SWEET algorithm, we present two additional examples: an irregular topography model with a strong velocity contrast and the IFP Marmousi model. We compare traveltimes and amplitudes computed by the SWEET algorithm for the Marmousi model with those obtained by using the time-domain finite-element solutions of the hyperbolic wave equation.

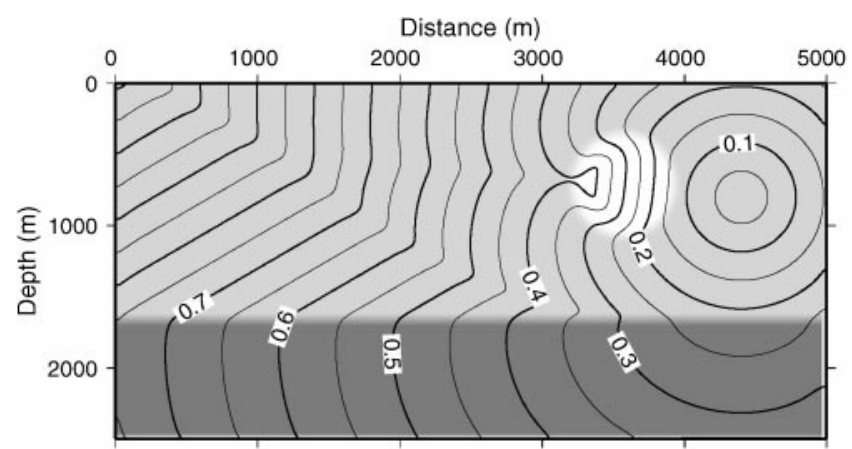

(a)

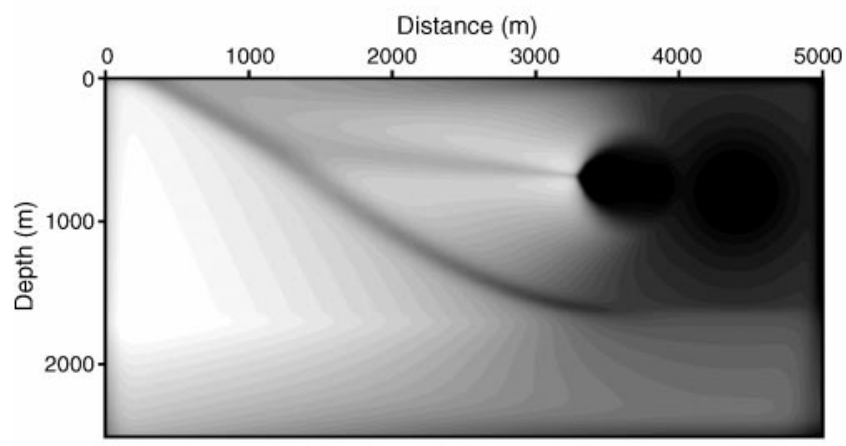

(b)

FIG. 6. (a) Traveltime contours and (b) a logarithmic amplitude image computed by the SWEET method for a two-layer model with a locally low velocity zone. The velocity is $4000 \mathrm{~m} / \mathrm{s}$ in the upper layer. The velocity is $2000 \mathrm{~m} / \mathrm{s}$ in the local low-velocity zone and $8000 \mathrm{~m} / \mathrm{s}$ in the lower layer.

\section{A two-layer model with a local low velocity zone}

We begin with a two-layer model that has high velocity variations (Figure 6a) and overplot the resulting traveltimes contoured with an interval of $0.05 \mathrm{~s}$. From Figure $6 \mathrm{a}$, we observe the shadow zone to the left of the low-velocity zone and the head waves in the upper layer. We conclude that the SWEET provides physically realistic, meaningful results even in the presence of a high velocity contrast. We also show corresponding logarithmic amplitudes in Figure 6b. We see two areas with low amplitude (indicated by white): one delineates the shadow zone behind the low-velocity inclusion and the other is associated with the head wave.

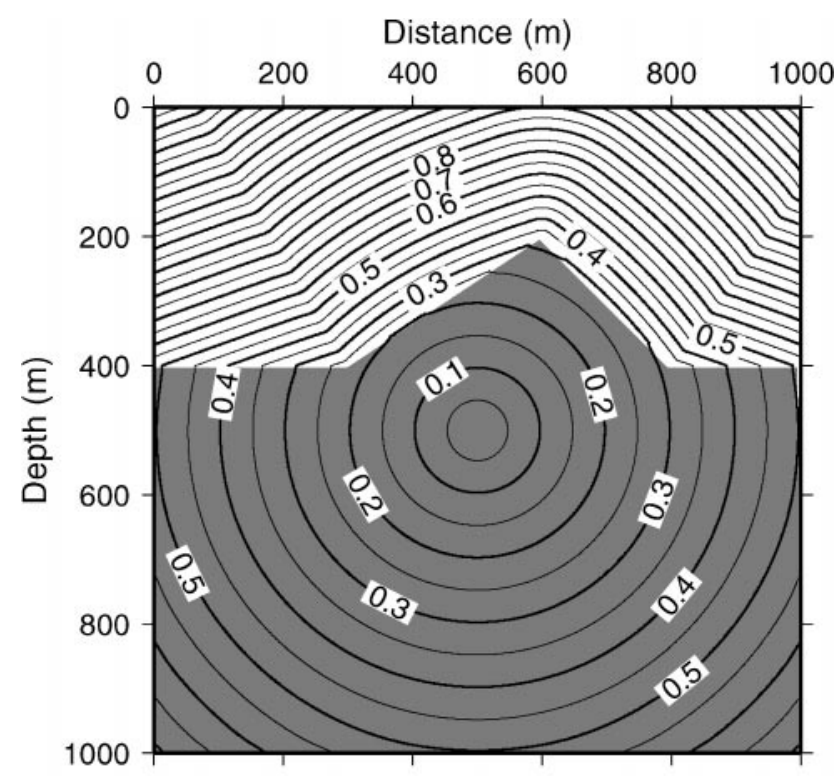

(a)

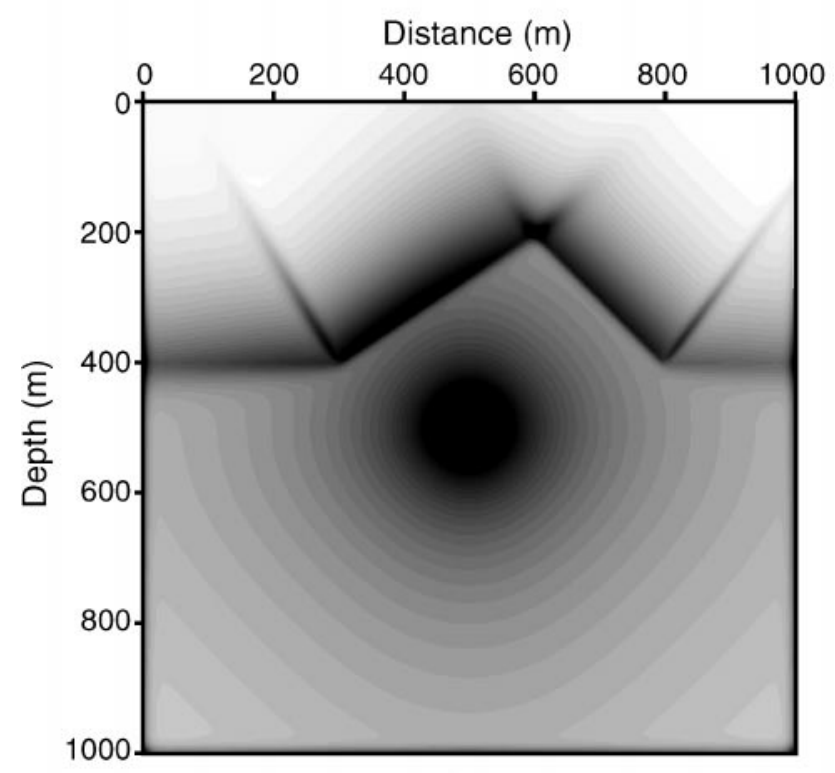

(b)

FIG. 7. (a) Traveltime contours and (b) an amplitude image obtained by the SWEET method for an irregular topography model. The velocity is $340 \mathrm{~m} / \mathrm{s}$ in the air and $1000 \mathrm{~m} / \mathrm{s}$ in the subsurface. 


\section{Irregular topography model}

To examine the stability of our traveltime and amplitude estimation in the presence of irregular topography, we take the model shown in Figure 7a. We assigned a velocity of $340 \mathrm{~m} / \mathrm{s}$ and a density of $0.00125 \mathrm{~g} / \mathrm{cm}^{3}$ to the air layer, and a velocity of $1000 \mathrm{~m} / \mathrm{s}$ and a density of $2.0 \mathrm{~g} / \mathrm{cm}^{3}$ in the subsurface. The grid spacing $\Delta x=\Delta z=2 \mathrm{~m}$. A source is located at the center of the model, at $x=500 \mathrm{~m}, z=500 \mathrm{~m}$.

Figures $7 \mathrm{a}$ and $7 \mathrm{~b}$ show traveltimes and amplitudes for the irregular topography model, respectively. The direct waves appear as circles in the subsurface. The waves, refracted towards the normal into the air, mimic the topography into the air. The amplitude decays faster in the air than in the subsurface, as shown in Figure $7 b$.

\section{The IFP Marmousi model}

Having successfully demonstrated the algorithmic stability in the two previous models, we proceed to test the accuracy for the IFP Marmousi model (Versteeg, 1994), shown in Figure 8a. The velocity varies from $1500 \mathrm{~m} / \mathrm{s}$ to $5500 \mathrm{~m} / \mathrm{s}$ in the Marmousi model. All the densities are fixed at $2.0 \mathrm{~g} / \mathrm{cm}^{3}$. The grid spacing $\Delta x=\Delta z=4 \mathrm{~m}$. We compare traveltimes obtained by our SWEET method (Figure 8a) with those picked from the syn- thetic seismograms generated by time-domain finite-element modeling (e.g., Marfurt, 1984) of the hyperbolic wave equation (Figure 8b). In the early times less than $1.5 \mathrm{~s}$, the SWEET method yields traveltimes compatible with time-domain finiteelement modeling solutions, but in the later times larger than $1.5 \mathrm{~s}$, we can observe slight differences between the traveltimes obtained by the SWEET method and the finite-element traveltimes. The differences mainly appear in the head waves. We presume that the differences originate from erroneously picking headwaves that have the characteristics of a smoothly emerging onset and a longer tail than other waves (Aki and Richards, 1980).

In Figures 9a and 9b, we superimpose traveltime contours calculated by the SWEET method at $0.8 \mathrm{~s}$ and $1.1 \mathrm{~s}$ on the snapshots computed by the finite-element solutions of the hyperbolic wave equation at $0.8 \mathrm{~s}$ and $1.1 \mathrm{~s}$, respectively. From Figure 9, we note that our traveltimes are in good agreement with the leading wavefronts of the snapshots.

In order to estimate the accuracy of the amplitudes computed by the SWEET method, we compare amplitudes of the first-arrival events obtained by the SWEET method with amplitudes computed from the synthetic seismograms obtained by finite-element modeling in Figure 10. We note that our amplitudes show a remarkable similarity to those of the finite-element solutions. Disagreements may arise

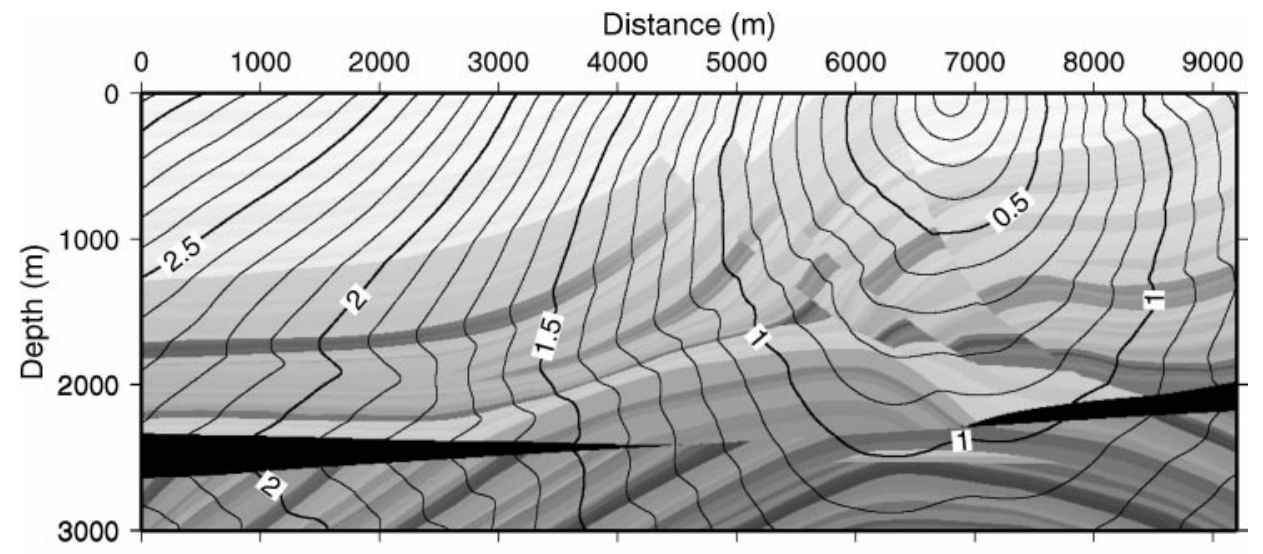

(a)

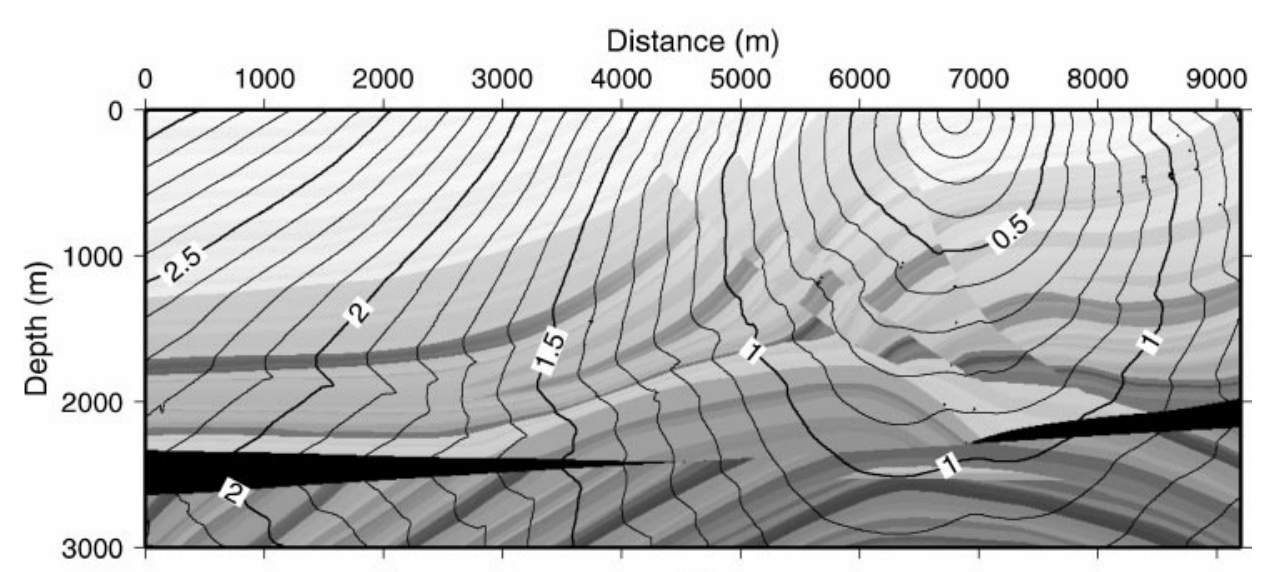

(b)

FIG. 8. Traveltime contours calculated by (a) the SWEET method and (b) finite-element modeling (FEM) for the Marmousi model. The velocities change from $1500 \mathrm{~m} / \mathrm{s}$ at the top of the model to $5500 \mathrm{~m} / \mathrm{s}$ at the bottom of the model. 
from errors in picking the amplitudes of the first-arrival events.

\section{CONCLUSIONS}

We presented the new SWEET method that computes traveltimes and amplitudes of first-arrival events for complex geological models for weighted prestack Kirchhoff depth migration. By solving the wave equation in the Laplace domain, we can suppress all the events with respect to the first-arrival event and thus easily compute the traveltime and amplitude of the first-arrival event. The accuracy of the SWEET method is sensitive to the Laplace frequency used to compute the traveltime and amplitude. By measuring errors between analytic traveltimes and numerical travel times obtained by the SWEET method for a simple, homogeneous model, we note that traveltime error is in inverse proportion to Laplace frequency with acceptable numerical-dispersion error. However, a large Laplace frequency requires a fine grid interval to minimize numerical dispersion, which leads to a higher computational cost. As a result, we need to select an optimum Laplace frequency that is large enough to effectively damp wave events following the first arrival while at the same time minimizing the numerical dispersion for a given velocity model. From our experience, with numerical dispersion error no larger than $0.4 \%\left(G^{\prime}=25\right)$, we can obtain accurate traveltimes. Any numerical algorithm (such as the frequency-domain finite-difference modeling technique) can exploit the SWEET feature. Higher order accurate finite-difference and finite-element modeling schemes will allow us to use coarser grids.

We showed that solving the Laplace-transformed hyperbolic wave equation is equivalent to simultaneously solving the eikonal and transport equations. Through numerical examples, we have showed that our SWEET method provides first-arrival traveltimes similar to traveltimes obtained from finite-element solutions of the eikonal equation but does not suffer from some of limitations encountered for high-velocity contrast. In addition, amplitudes computed by the SWEET algorithm are not adversely affected by caustics.

This implementation of our SWEET method only computes first-arrival rather than maximum-energy arrival time. Our next task is to exploit this estimate of first arrivals to define a window in which we search for a later maximum energy event. The SWEET method can easily be extended to any algorithm that can be implemented in the frequency domain, including 3-D topographic models.

\section{ACKNOWLEDGMENT}

This work was financially supported by National Research Laboratory Project of the Korea Ministry of Science and Technology, Brain Korea 21 project of the Korea Ministry of

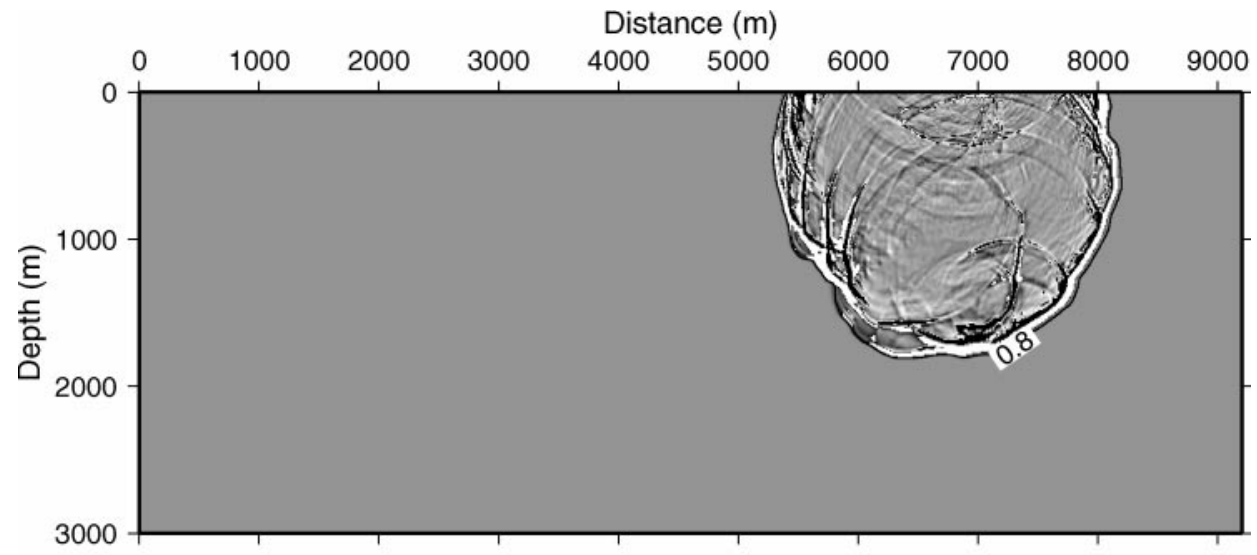

(a)

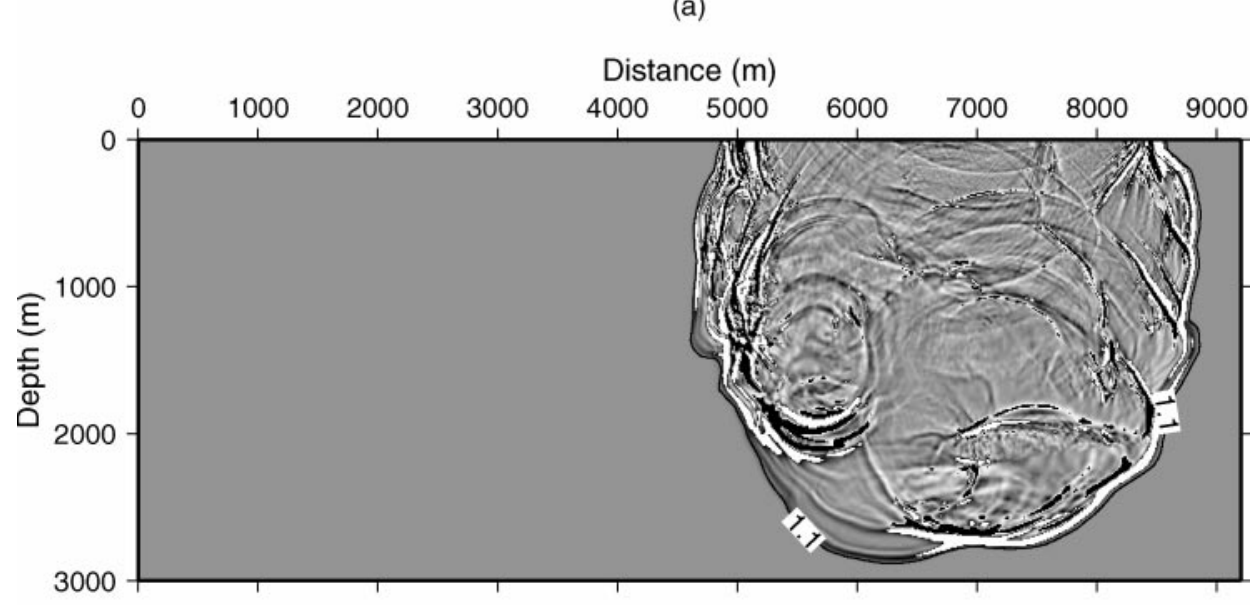

(b)

FIG. 9. Traveltime contours computed by the SWEET method for the Marmousi model at (a) $0.8 \mathrm{~s}$ and (b) $1.1 \mathrm{~s}$ overlaid on snapshot image obtained by an FEM algorithm. 


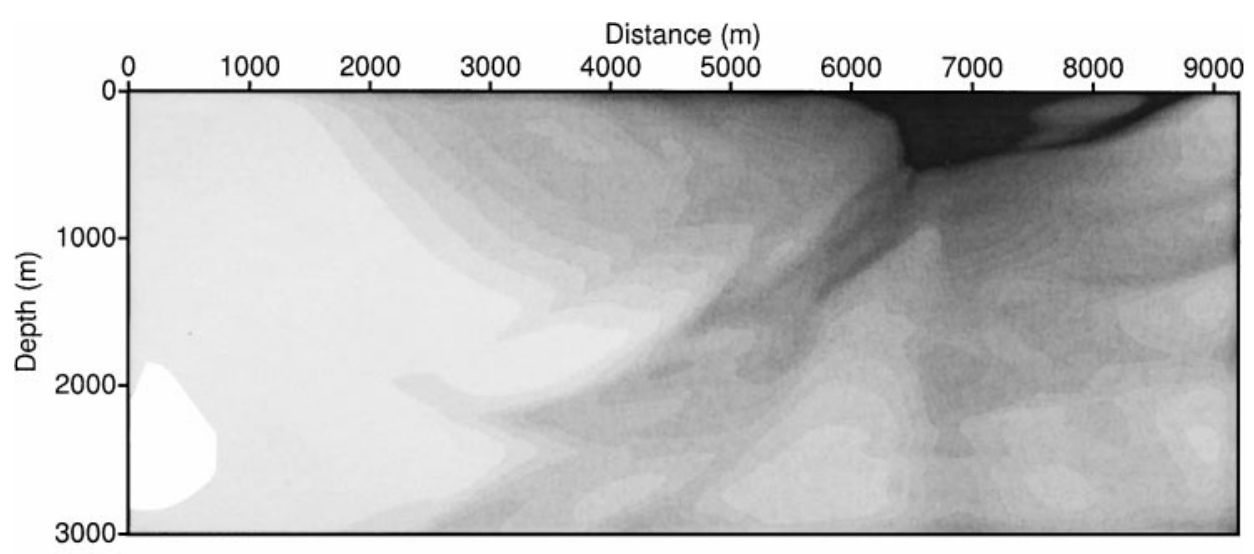

(a)

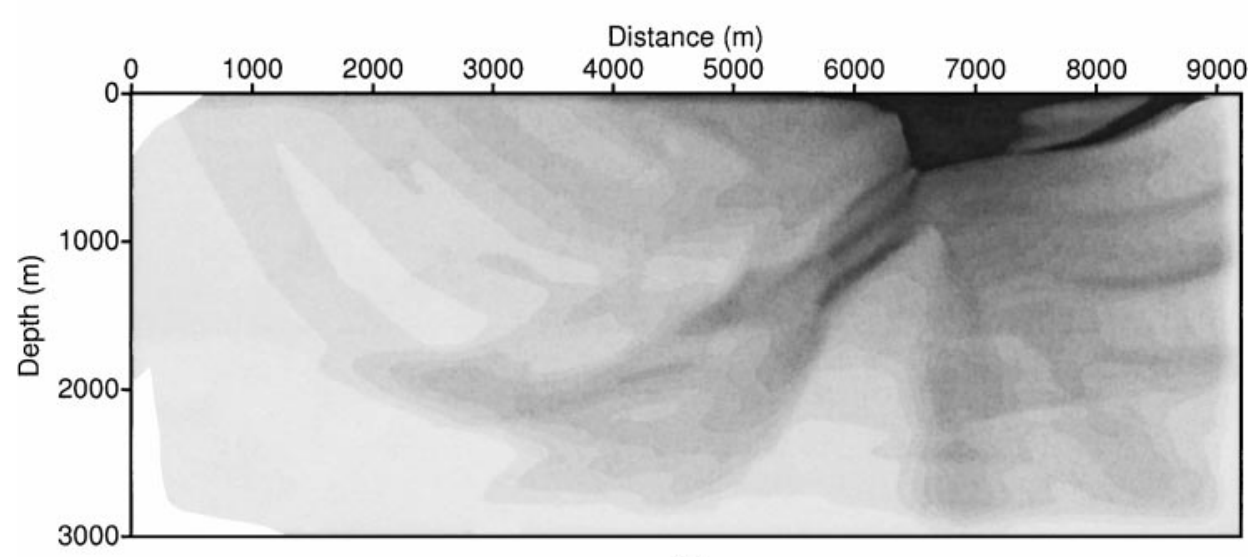

(b)

FIG. 10. Amplitude images calculated by (a) the SWEET method and (b) the FEM modeling technique for the Marmousi model.

Education, grant No. R05-2000-00003 from the Basic Research Program of the Korea Science \& Engineering Foundation, and grant No. PM10300 from Korea Ocean Research \& Development Institute.

\section{REFERENCES}

Aki, K., and Richards, P. G., 1980, Quantitative seismology: Theory and Methods: W. H. Freeman \& Co.

Cerveny, V., Molotkov, I. A., and Psencik, I., 1977, Ray method in seismology: Univ. of Karlova Press.

Coultrip, R. L., 1993, High-accuracy wavefront tracing traveltime calculation: Geophysics, 58, 284-292.

Ettrich, N., and Gajewski, D., 1998, Traveltime computation by perturbation with FD-eikonal solvers in isotropic and weakly anisotropic media: Geophysics, 63, 1066-1078.

Kelly, K. R., and Marfurt, K. J., 1990, Numerical modeling of seismic wave propagation: Soc. Expl. Geophys.

Jo, C. H. Shin, C. and Suh, J. H. 1996, An optimal 9-point, finite difference, frequency-space, 2-D scalar wave extrapolator: Geophysics, 61, 529-537.

Kreyszig, E., 1993, Advanced engineering mathematics, 7th ed.: John Wiley and Sons, Inc.

Marfurt, K. J. 1984, Accuracy of finite-difference and finite-element modeling of the scalar and elastic wave equations: Geophysics, 49, $533-549$.
Nichols, D. E., 1996, Maximum energy traveltimes calculated in the seismic frequency band: Geophysics, 61, 253-263.

Podvin, P., and Lecomte, I., 1991, Finite-difference computation of traveltimes in very contrasted velocity models: A massively parallel approach and its associated tools: Geophys. J. Internat., 105, 271-284

Qin, F., Luo, Y., Olsen, K. B., Cai, W. and Schuster, G. T., 1992, Finitedifference solution of the eikonal equation along expanding wave fronts: Geophysics, 57, 478-487.

Schneider Jr., W. A., Ranzinger, K. A., Balch, A. H., and Kruse, C. 1992, A dynamic programming approach to first-arrival traveltime computation in media with arbitrarily distributed velocities: Geophysics, 57, 39-50.

Shin, S., Shin, C., and Kim, W., 2000, Traveltime calculation using monochromatic one way wave equation: 70th Ann. Internat. Mtg., Soc. Expl. Geophys., Expanded Abstracts, 2313-2316.

Thurber, C. H., and Ellsworth, W. C, 1980, Rapid solution of ray tracing problems in heterogeneous media: Bull. Seis. Soc. Am., 70, 11381148 .

van Trier, J., and Symes, W. W., 1991, Upwind finite-difference computation of traveltimes: Geophysics, 56, 812-821.

Versteeg, R., 1994, The Marmousi experience: Velocity model determination on a synthetic complex data set: The Leading Edge, 13, 927-936.

Vidale, 1988, Finite-difference calculation of traveltimes: Bull. Seis. Soc. Am., 78, 2062-2076.

Vinje, V., Iversen, E., and Gjoystdal H., 1993, Traveltime and amplitude estimation using wave front construction: Geophysics, 58, 1157 1166. 


\section{APPENDIX A}

\section{THE DERIVATIVE WAVEFIELD}

To obtain the derivative wavefield $(\partial \tilde{\mathbf{U}} / \partial s)$, we differentiate equation (3) with respect to the Laplace frequency $s$ :

$$
\left[\mathbf{M} s^{2}+\mathbf{K}\right] \frac{\partial \tilde{\mathbf{U}}}{\partial s}=\tilde{\mathbf{F}}^{*}
$$

with

$$
\tilde{\mathbf{F}}^{*}=-\mathbf{2 s M} \tilde{\mathbf{U}}
$$

Equation (A-1) is obtained by replacing $\tilde{\mathbf{U}}$ and $\tilde{\mathbf{F}}$ by $(\partial \tilde{\mathbf{U}} / \partial s)$ and $\tilde{\mathbf{F}}^{*}$, respectively, in equation (3). Since $\tilde{\mathbf{F}}$ is a constant in equation (3), the derivative of $\tilde{\mathbf{F}}$ is zero. The vector $\tilde{\mathbf{F}}^{*}$ becomes a new source used to compute the derivative of the wavefield $(\tilde{\mathbf{U}})$ with respect to the Laplace frequency. Once we factor the real impedance matrix and obtain the wavefield $(\tilde{\mathbf{U}})$ in the Laplace domain, the computation of the derivative wavefield $(\partial \tilde{\mathbf{U}} / \partial s)$ only requires one more forward and backward substitution.

\section{THE TRANSPORT AND EIKONAL EQUATIONS}

A 2-D scalar wave equation is given in the time domain by

$$
\nabla^{2} u=\frac{1}{v^{2}} \frac{\partial^{2} u}{\partial t^{2}}
$$

where $v$ is the velocity and $u$ is the wavefield. Taking the Laplace transform of equation (B-1) gives

$$
\nabla^{2} \tilde{U}-\frac{s^{2}}{v^{2}} \tilde{U}=0
$$

The suppressed wavefield $\tilde{U}$ is given in the Laplace domain as

$$
\tilde{U}(s)=A e^{-s \tau}, \tau=\tau(x, y, z),
$$

where $A$ and $\tau$ are the amplitude and the traveltime of the first arrival.

In the high-frequency limit, substituting equation (B-3) into equation (B-2) gives

$$
s^{2} A\left\{(\nabla \tau)^{2}-\frac{1}{v^{2}}\right\}-s\left\{2 \nabla A \nabla \tau+A \nabla^{2} \tau\right\}+\nabla^{2} A=0 .
$$

The first term in braces in equation (B-4) when equated to zero gives the eikonal equation and the second term in braces gives the transport equation. Thus by using the SWEET method, we can simultaneously solve both the transport and the eikonal equation.

APPENDIX C

\section{DISPERSION RELATION}

Since there is no simple relationship between the Laplace frequency and temporal frequency, we cannot use the classical von Neumann analysis based on monofrequency plane waves. Instead, we note that dispersion is directly related to the eigenvalues, $\omega^{2}$, of the discrete system (Kelly and Marfurt, 1990, 516-520). We therefore express the dispersion relation of the Laplace-transformed wave equation as the square root of the ratio of numerical eigenvalue to analytic eigenvalue. We follow the von Neumann analysis by defining an eigenfunction as $e^{k_{x} x+k_{z} z}$ with $k_{x}=k \cos \theta$ and $k_{z}=k \sin \theta$. If we substitute this eigenfunction into the Laplace-transformed wave equation (B-2), we obtain a relationship

$$
k_{x}^{2}+k_{z}^{2}=k^{2}=\frac{s^{2}}{v^{2}},
$$

where $s^{2}$ is the analytic eigenvalue. The numerical eigenvalue is obtained by substituting the eigenfunction $e^{ \pm\left(k_{x} x+k_{z} z\right)}$ into the discrete formula extracted from equation (3). The traditional finite-element method does not generate computational stencils but directly constructs assembled stiffness and mass matrices (Marfurt, 1984). Nevertheless, a computational stencil can be extracted from the assembled matrices. The discrete equation is expressed for the lumped mass-matrix operator as

$$
\begin{aligned}
\frac{s^{2}}{v^{2}} \Delta x \Delta z u_{i, j}= & \frac{1}{6} \frac{\Delta z}{\Delta x}\left[u_{i-1, j-1}-2 u_{i, j-1}+u_{i+1, j-1}\right] \\
& +\frac{2}{3} \frac{\Delta z}{\Delta x}\left[u_{i-1, j}-2 u_{i, j}+u_{i+1, j}\right] \\
& +\frac{1}{6} \frac{\Delta z}{\Delta x}\left[u_{i-1, j+1}-2 u_{i, j+1}+u_{i+1, j+1}\right] \\
& +\frac{1}{6} \frac{\Delta x}{\Delta z}\left[u_{i-1, j-1}-2 u_{i-1, j}+u_{i-1, j+1}\right] \\
& +\frac{2}{3} \frac{\Delta x}{\Delta z}\left[u_{i, j-1}-2 u_{i, j}+u_{i, j+1}\right] \\
& +\frac{1}{6} \frac{\Delta x}{\Delta z}\left[u_{i+1, j-1}-2 u_{i+1, j}+u_{i+1, j+1}\right] .
\end{aligned}
$$

The dispersion relation $R$ obtained by substituting the eigenfunction into the above discrete equation is written as

$$
R(s, v, \theta, \Delta)=\sqrt{\left(\frac{A}{B}\right) /\left(\frac{s^{2}}{v^{2}}\right)}
$$

with 


$$
\begin{aligned}
A= & {\left[\frac{1}{6} e^{-k_{z} \Delta z}+\frac{2}{3}+\frac{1}{6} e^{k_{z} \Delta z}\right]\left[e^{-k_{x} \Delta x}-2+e^{k_{x} \Delta x}\right] } \\
& +\left[e^{-k_{z} \Delta z}-2+e^{k_{z} \Delta z}\right]\left[\frac{1}{6} e^{-k_{x} \Delta x}+\frac{2}{3}+\frac{1}{6} e^{k_{x} \Delta x}\right],
\end{aligned}
$$

$$
B=\Delta x \Delta z
$$

and

$$
\Delta=\Delta x=\Delta z
$$

where $\left(v^{2} A / B\right)$ is the numerical eigenvalue. Since numerical dispersion is dependent upon the Laplace frequency $s$, velocity $v$, propagation angle $\theta$, and grid interval $\Delta$, we can determine an optimal Laplace frequency to suppress all wave events with respect to the first-arrival event while minimizing numerical dispersion with respect to velocity, propagation angle, and grid interval.

For comparison, we also present the dispersion relation for the frequency-domain wave equation. The frequency-domain discrete equation for the lumped mass-matrix operator is obtained by replacing $s^{2}$ by $-\omega^{2}$ in equation (C-2). In this case, the analytic eigenvalue is $\omega^{2}$ and the eigenfunction is $e^{ \pm i\left(k_{x} x+k_{z} z\right)}$ with $k_{x}=k \cos \theta, k_{z}=k \sin \theta$, and $k=\omega / v$. As a result, the frequency-domain dispersion relation is expressed as

$$
R(\omega, v, \theta, \Delta)=\sqrt{\left(\frac{C}{D}\right) /\left(-\frac{\omega^{2}}{v^{2}}\right)}
$$

with

$$
\begin{aligned}
C= & {\left[\frac{1}{6} e^{-i k_{z} \Delta z}+\frac{2}{3}+\frac{1}{6} e^{i k_{z} \Delta z}\right]\left[e^{-i k_{x} \Delta x}-2+e^{i k_{x} \Delta x}\right] } \\
& +\left[e^{-i k_{z} \Delta z}-2+e^{i k_{z} \Delta z}\right]\left[\frac{1}{6} e^{-i k_{x} \Delta x}+\frac{2}{3}+\frac{1}{6} e^{i k_{x} \Delta x}\right]
\end{aligned}
$$

and

$$
D=\Delta x \Delta z
$$

In the frequency domain, we determine the number of gridpoints per wavelength by using

$$
G=\frac{v_{\text {min }}}{f_{\text {optimum }} \Delta}=\frac{2 \pi v_{\text {min }}}{\omega_{\text {optimum }} \Delta} .
$$

Equation (C-10) is similar to equation (12) in the Laplace domain. We compare the dispersion relation for the Laplacedomain wave equation with that of the frequency-domain wave equation in Table C-1.

Table C-1. Comparison of the dispersion relations for the Laplace and the frequency-domain wave equation.

\begin{tabular}{lcc}
\hline & Frequency domain & Laplace domain \\
\hline Eigenvalue & $\omega^{2}$ & $s^{2}$ \\
Eigenfunction & $e^{i\left(k_{x} x+k_{z} z\right)}$ & $e^{k_{x} x+k_{z} z}$ \\
Optimum frequency & $\omega_{\text {optimum }}=\frac{2 \pi v_{\min }}{G \Delta}$ & $s_{\text {optimum }}=\frac{2 \pi v_{\text {ave }}}{G^{\prime} \Delta}$ \\
Within $0.4 \%$ errors & $G=25$ & $G^{\prime}=25$ \\
\hline
\end{tabular}

